\title{
The order of birational rowmotion
}

\author{
Darij Grinberg]| $\mid$ and Tom Roby $\|^{2}$ \\ ${ }^{1}$ Massachusetts Institute of Technology, Cambridge, MA 02139, USA \\ ${ }^{2}$ University of Connecticut, Storrs, CT 06269, USA
}

\begin{abstract}
.
Various authors have studied a natural operation (under various names) on the order ideals (equivalently antichains) of a finite poset, here called rowmotion. For certain posets of interest, the order of this map is much smaller than one would naively expect, and the orbits exhibit unexpected properties. In very recent work (inspired by discussions with Berenstein) Einstein and Propp describe how rowmotion can be generalized: first to the piecewise-linear setting of order polytopes, then via detropicalization to the birational setting.

In the latter setting, it is no longer a priori clear even that birational rowmotion has finite order, and for many posets the order is infinite. However, we are able to show that birational rowmotion has the same order, $p+q$, for the poset $P=[p] \times[q]$ (product of two chains), as ordinary rowmotion. We also show that birational (hence ordinary) rowmotion has finite order for some other classes of posets, e.g., the upper, lower, right and left halves of the poset above, and trees having all leaves on the same level. Our methods are based on those used by Volkov to resolve the type $A A$ (rectangular) Zamolodchikov Periodicity Conjecture.
\end{abstract}

\section{Résumé.}

Une opération naturelle sur les idéaux d'ordre (ou, de façon équivalente, sur les antichaînes) d'un ensemble partiellement ordonné fini, appelée ici rowmotion, a été étudiée par divers auteurs. Pour certains ensembles partiellement ordonnés particulièrement intéressants, l'ordre de cette application rowmotion est beaucoup plus petit que ce à quoi on peut s'attendre naïvement, et ses orbites ont des propriétés inattendues. Dans un travail très récent (inspiré par des conversations avec Berenstein), Einstein et Propp ont décrit comment le rowmotion peut être généralisé: d'abord au contexte linéaire par morceaux des polytopes d'ordre, ensuite au contexte birationnel par détropicalisation.

Dans ce dernier contexte, il n'est même plus clair a priori si le rowmotion birationnel est d'ordre fini, et son ordre est infini pour beaucoup d'ensembles partiellement ordonnés. Malgré tout, nous avons pu montrer que le rowmotion birationnel a le même ordre $p+q$ pour l'ensemble partiellement ordonné $P=[p] \times[q]$ (produit de deux chaînes) que le rowmotion ordinaire. Nous montrons aussi que le rowmotion birationnel (et par conséquent le rowmotion ordinaire aussi) est d'ordre fini pour d'autres classes d'ensembles partiellement ordonnés, par exemple les moitiés supérieure, inférieure, droite et gauche de l'ensemble partiellement ordonné mentionné auparavant, et les arbres dont toutes les feuilles sont au même niveau. Nos méthodes sont basées sur celles utilisées par Volkov pour établir la conjecture de périodicité de Zamolodchikov de type $A A$ (rectangulaire).

Keywords: antichains, birational actions, birational rowmotion, Brouwer-Schrijver map, cluster algebras, orbit, order ideals, Panyushev complementation, poset, product of chains, promotion, rowmotion, toggle group, Zamolodchikov conjecture.

*Partially supported by NSF Grant \#1001905

$\dagger$ Partially supported by NSF Grant \#1001905

1365-8050 @ 2014 Discrete Mathematics and Theoretical Computer Science (DMTCS), Nancy, France 


\section{Introduction}

Numerous authors have studied a natural operation (under various names) on the order ideals (equivalently antichains) of a finite poset, here called rowmotion, following Striker and Williams [StWi11]. For certain posets of interest, the order of this map is much smaller than one would naively expect [BrSchr74], and its orbits exhibit further unexpected patterns as found in [PrRo13], [Pan08] and [AST11].

In very recent work, Einstein and Propp [EiPr13] describe how rowmotion can be generalized: first to the piecewise-linear setting of an order polytope [Stan86, Definition 1.1], then via detropicalization to the birational setting; they also find applications to classical combinatorics of tableaux. Much of their paper focuses on the case where the poset $P$ is $[p] \times[q]$, a product of two chains, generalizing earlier work of Propp and Roby in the combinatorial setting [PrRo13]. Their paper provides much of the context and the motivation for our work.

In the birational setting, it is no longer a priori clear even that rowmotion has finite order, since the set of possible labellings of $P$ is infinite (unlike the set of antichains or order ideals). Indeed, for some simple posets (including the product of three length-2 chains) the order is infinite. However, we prove as one of our main results that birational rowmotion has the same order, $p+q$, for the poset $P=[p] \times[q]$ as ordinary rowmotion. We have also found that birational rowmotion has finite order for several other classes of posets, e.g., the upper, lower, right and left halves of the poset above, and trees which are graded (as posets). Our methods are based on those used by Volkov to resolve the type $A A$ (rectangular) Zamolodchikov Periodicity Conjecture [Volk06]. In the case of a rectangle, we also demonstrate a symmetry property of birational rowmotion conjectured by James Propp and Tom Roby.

In this extended abstract, proofs are only roughly outlined. Full arguments and further details can be found in [GrRo13]. Applications of our results (specifically Theorems 33] and 34] are found in [EiPr13].

\subsection{Acknowledgments}

The authors are grateful to James Propp for many helpful suggestions guiding this work and explaining his own; to Arkady Berenstein for instruction how to generalize rowmotion to the birational setting; to Gregg Musiker and Pavlo Pylyavskyy for suggesting the link to Zamolodchikov's conjecture, and references to Volkov's paper; to David Einstein for regular communications and many helpful suggestions; to Jessica Striker and Nathan Williams for helping understand their work and some background notions; to Hugh Thomas and the referees for improvements on the writing.

\section{Birational Rowmotion}

Except when stated otherwise, we follow the standard notations and conventions for posets of Stanley [Stan11, Ch. 3] (but we write our maps on the left). We recall one definition of classical rowmotion ([StWi11, Theorem 4.4], originally due to Cameron and Fon-der-Flaass):

Definition 1. Let $P$ be a finite poset, and $J(P)$ the set of the order ideals of $P$. (Classical) rowmotion is a map $J(P) \rightarrow J(P)$ which can be defined in various ways, perhaps the simplest being as follows: For every $v \in P$, let $\mathbf{t}_{v}: J(P) \rightarrow J(P)$ be the map sending every order ideal $S \in J(P)$ to 
$\left\{\begin{array}{l}S \cup\{v\}, \text { if } v \notin P \text { and } S \cup\{v\} \in J(P) ; \\ S \backslash\{v\}, \text { if } v \in P \text { and } S \backslash\{v\} \in J(P) ; \\ S, \text { otherwise }\end{array}\right.$

Let $\left(v_{1}, v_{2}, \ldots, v_{m}\right)$ be any linear extension of $P$. Rowmotion is then defined as the composition $\mathbf{t}_{v_{1}} \circ$ $\mathbf{t}_{v_{2}} \circ \ldots \circ \mathbf{t}_{v_{m}}: J(P) \rightarrow J(P)$; this is independent of the linear extension chosen.

For most of this abstract we will study an analogue of this map in the birational setting; Definition 1 will only be used in Section 6. Let us now introduce the setting we will be working in ([GrRo13, §1- 33$])$.

For any poset $P$ we write $u \lessdot v$ if $u$ is covered by $v$, i.e., if we have have $u<v$ and there is no $w \in P$ such that $u<w<v$; similarly we use $u>v$ for the dual relation " $u$ covers $v$ ". Define $\widehat{P}$ to be the poset $P$ with the addition of a minimum element 0 and a maximum element 1 (even if such elements already exist within $P$ itself; in other words, $\widehat{P}$ is the ordinal sum $\{0\} \oplus P \oplus\{1\})$.

Assume $P$ is a graded poset, in the sense that every maximal chain in $P$ has the same length (say $n-1)$. Then $\widehat{P}$ is also a graded poset; for every $v \in \widehat{P}$ we define the degree (deg) of $v$ to be the maximum length (= cardinality minus one) of a chain in $\widehat{P}$ with highest element $v$. This agrees with the ordinary notion of rank in $\widehat{P}$, but is off by 1 in $P$ itself. We say that $P$ is $n$-graded if the degree of $1 \in \widehat{P}$ is $n+1$. For each $i \in\{0,1,2, \ldots, n+1\}$, let $\widehat{P}_{i}$ denote $\{v \in \widehat{P}: \operatorname{deg} v=i\}$.

Definition 2. Let $P$ be a poset. We fix an infinite field $\mathbb{K}$ for the rest of this abstract. A $\mathbb{K}$-labelling of $P$ will mean a map $f: \widehat{P} \rightarrow \mathbb{K}$. Thus, $\mathbb{K}^{\widehat{P}}$ is the set of all $\mathbb{K}$-labellings of $P$. If $f$ is a $\mathbb{K}$-labelling of $P$ and $v$ is an element of $\widehat{P}$, then $f(v)$ will be called the label of $f$ at $v$.

Most of the maps that we will be considering are rational maps between algebraic varieties (specifically, between open subsets of affine or projective spaces). We use the punctured arrow $\rightarrow-\rightarrow$ to signify rational maps (i.e., a rational map from a variety $U$ to a variety $V$ is called a rational map $U \rightarrow-\rightarrow$ ). A rational map $U \rightarrow V$ is said to be dominant if its image is dense in $V$ (with respect to the Zariski topology).

Definition 3. Let $v \in P$. We define a rational map $T_{v}: \mathbb{K}^{\widehat{P}} \rightarrow \mathbb{K}^{\widehat{P}}$ called the (birational) $v$-toggle by

$$
\left(T_{v} f\right)(w)= \begin{cases}f(w), & \text { if } w \neq v ; \\ \frac{1}{f(v)} \cdot\left(\sum_{\substack{u \in \widehat{P} ; \\ u \ll v}} f(u)\right)\left(\sum_{\substack{u \in \widehat{P} ; \\ u \gg v}} \frac{1}{f(u)}\right)^{-1}, \quad \text { if } w=v \quad \text { for all } w \in \widehat{P}\end{cases}
$$

for all $f \in \mathbb{K}^{\widehat{P}}$ which don't render one of the denominators above zero.

It is very easy to check that the maps $T_{v}$ are involutive $\left(T_{v}^{2}=\mathrm{id}\right)$ dominant rational maps and satisfy the following "locality principle":

Proposition 4. Let $P$ be a finite poset. Let $v \in P$ and $w \in P$. Then, $T_{v} \circ T_{w}=T_{w} \circ T_{v}$, unless we have either $v \lessdot w$ or $w \lessdot v$.

Combining this with [AKSch12, Prop. 4.1] (the transposition case), we obtain:

Corollary 5. Let $P$ be a finite poset. Let $\left(v_{1}, v_{2}, \ldots, v_{m}\right)$ be a linear extension of $P$. Then, the dominant rational map $T_{v_{1}} \circ T_{v_{2}} \circ \ldots \circ T_{v_{m}}: \mathbb{K}^{\widehat{P}} \rightarrow \mathbb{K}^{\widehat{P}}$ is well-defined and independent of the choice of the linear extension $\left(v_{1}, v_{2}, \ldots, v_{m}\right)$.

Definition 6. Let $P$ be a finite poset. Birational rowmotion is defined as the dominant rational map $R:=T_{v_{1}} \circ T_{v_{2}} \circ \ldots \circ T_{v_{m}}: \mathbb{K}^{\widehat{P}}-\rightarrow \mathbb{K}^{\widehat{P}}$, where $\left(v_{1}, v_{2}, \ldots, v_{m}\right)$ is a linear extension of $P$. 
The reason for the names "birational toggle" and "birational rowmotion" is explained in the paper [EiPr13], in which birational rowmotion (there defined over $\mathbb{R}_{+}$) is denoted by $\rho_{\mathcal{B}}$.

Example 7. Let $P=[2] \times[2]$, with generic initial labeling $f$ as shown (with $(2,1)$ being the leftmost element), except that we also assign $f(\hat{0})=f(\hat{1})=1$ in $\widehat{P}$. Then applying the toggling operators $T_{(2,2)}$, $T_{(2,1)}, T_{(1,2)}, T_{(1,1)}$ in that order (as shown) results in the final labeling $R f$.

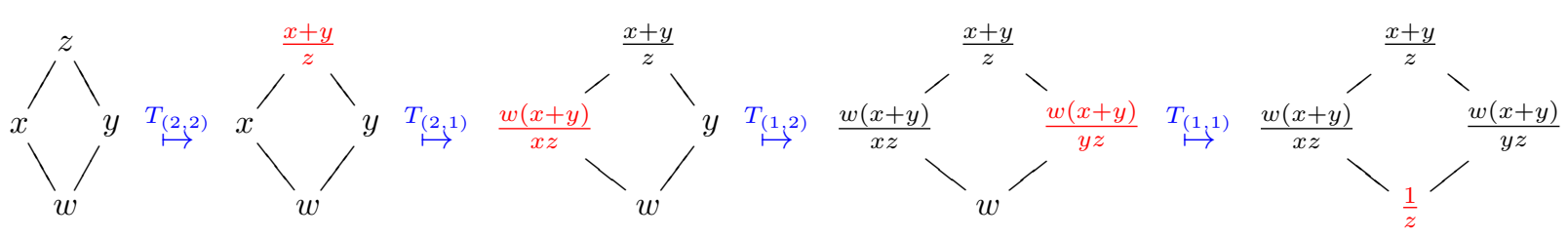

If we apply the birational rowmotion operator four times, we return to our initial labeling:

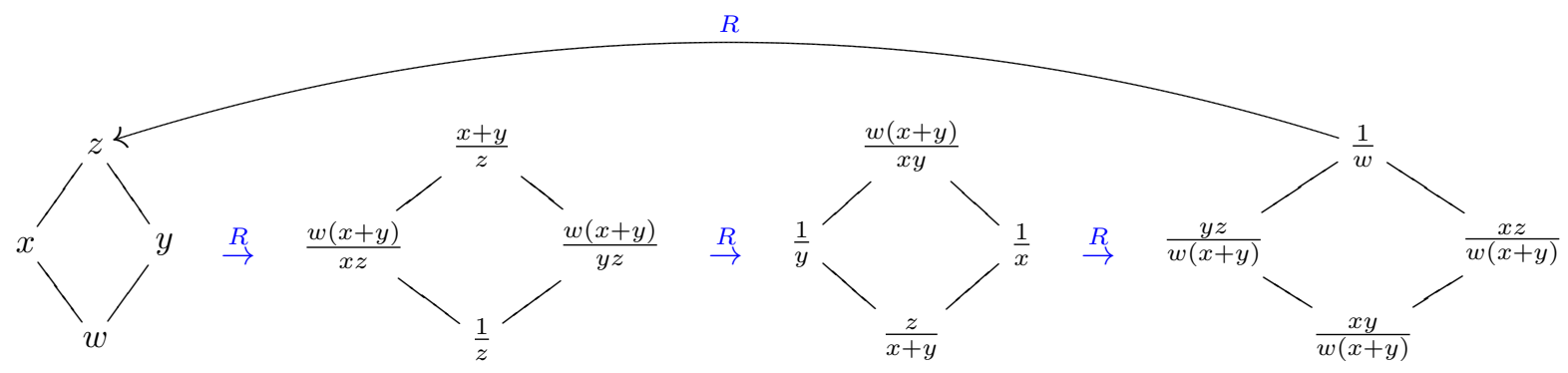

Doing toggles in the opposite order does not give anything new, just the inverse of the above map:

Proposition 8. Let $P$ be a finite poset. Then, birational rowmotion $R$ is invertible (as a rational map). Its inverse $R^{-1}$ is $T_{v_{m}} \circ T_{v_{m-1}} \circ \ldots \circ T_{v_{1}}: \mathbb{K}^{\widehat{P}} \rightarrow \mathbb{K}^{\widehat{P}}$, where $\left(v_{1}, v_{2}, \ldots, v_{m}\right)$ is a linear extension of $P$.

Although $R$ is defined for any finite poset, we will only consider it here for graded posets. In this case the rowmotion map can be factored as a product of simpler involutions:

Definition 9. Let $P$ be an $n$-graded poset. Let $i \in\{1,2, \ldots, n\}$. Then, let $R_{i}$ denote the dominant rational map $T_{u_{1}} \circ T_{u_{2}} \circ \ldots \circ T_{u_{k}}: \mathbb{K}^{\widehat{P}} \rightarrow \mathbb{K}^{\widehat{P}}$, where $\left(u_{1}, u_{2}, \ldots, u_{k}\right)$ is any list of the elements of $\widehat{P}_{i}$ with every element of $\widehat{P}_{i}$ appearing exactly once in the list. This map $T_{u_{1}} \circ T_{u_{2}} \circ \ldots \circ T_{u_{k}}$ is well-defined (in particular, it does not depend on the list $\left.\left(u_{1}, u_{2}, \ldots, u_{k}\right)\right)$.

Remark 10. Let $P$ be an n-graded poset. Then, $R=R_{1} \circ R_{2} \circ \ldots \circ R_{n}$. Moreover, each $R_{i}$ is an involution (that is, $R_{i}^{2}=\mathrm{id}$ on the set where $R_{i}$ is defined).

\section{The $\mathbf{w}_{i}(f)$ checksums}

Definition 11. Let $P$ be an $n$-graded poset. Let $f \in \mathbb{K}^{\widehat{P}}$. Let $i \in\{0,1, \ldots, n\}$. Then, $\mathbf{w}_{i}(f)$ will denote the element of $\mathbb{K}$ defined by

$$
\mathbf{w}_{i}(f)=\sum_{\substack{x \in \widehat{P}_{i} ; y \in \widehat{P}_{i+1} ; \\ y \gg x}} \frac{f(x)}{f(y)} .
$$


(This element is not always defined, but is defined in the "generic" case when $0 \notin f(\widehat{P})$.)

The intuition behind this definition is that the $\mathbf{w}_{i}(f)$ is a kind of "checksum" for the labelling $f$ which displays how much its labels at degree $i+1$ differ from those at degree $i$. These "checksums" alone are not very informative, but combined with the so-called homogenization of $f$ (which will be introduced in Definition 14 and the value $f(0)$ they will suffice to uniquely determine $f$, thus helping us "split up" the dynamical system of a labelling under $R$ into three subsystems. As for now let us see that they behave in a rather simple way under the maps $R$ and $R_{j}$. Indeed, simple computations ([GrRo13, §4]) show:

Proposition 12. Let $P$ be an $n$-graded poset. Let $i \in\{1,2, \ldots, n\}$. Then, every $f \in \mathbb{K}^{\widehat{P}}$ satisfies

$$
\begin{aligned}
& \left(\mathbf{w}_{0}\left(R_{i} f\right), \mathbf{w}_{1}\left(R_{i} f\right), \ldots, \mathbf{w}_{n}\left(R_{i} f\right)\right) \\
& =\left(\mathbf{w}_{0}(f), \mathbf{w}_{1}(f), \ldots, \mathbf{w}_{i-2}(f), \mathbf{w}_{i}(f), \mathbf{w}_{i-1}(f), \mathbf{w}_{i+1}(f), \mathbf{w}_{i+2}(f), \ldots, \mathbf{w}_{n}(f)\right) .
\end{aligned}
$$

From Proposition 12, and Remark 10 we conclude:

Proposition 13. Let $P$ be an $n$-graded poset. Then, every $f \in \mathbb{K}^{\widehat{P}}$ satisfies

$$
\left(\mathbf{w}_{0}(R f), \mathbf{w}_{1}(R f), \ldots, \mathbf{w}_{n}(R f)\right)=\left(\mathbf{w}_{n}(f), \mathbf{w}_{0}(f), \mathbf{w}_{1}(f), \ldots, \mathbf{w}_{n-1}(f)\right) \text {. }
$$

\section{Homogeneous labellings}

In general, birational rowmotion $R$ has an "avalanche effect", meaning that a change of just one label in a labelling will often "spread" through a large part of the poset when $R$ is applied; the effect of such a change is hard to track in general. However, for a graded poset $P$, if we simultaneously multiply the labels at all vertices of a given degree with a given scalar, then the changes this causes to the behavior of the labelling under $R$ are rather predictable. Explicit formulas are given in [GrRo13, §5]; here we content ourselves with introducing a notion of homogeneous equivalence which formalizes the idea of a "labelling modulo scalar factors at each degree" ([GrRo13, §6]). (Our definition is superficially different from the one given in [GrRo13, §6], but easily seen to be equivalent.)

Definition 14. Let $P$ be an $n$-graded poset.

(a) A $\mathbb{K}$-labelling $f \in \mathbb{K}^{\widehat{P}}$ is said to be zero-free if for every $i \in\{0,1, \ldots, n+1\}$, there exists some $v \in \widehat{P}_{i}$ satisfying $f(v) \neq 0$. Let $\mathbb{K}_{\neq 0}^{\widehat{P}}$ be the set of all zero-free $\mathbb{K}$-labellings. Clearly, this set $\mathbb{K}_{\neq 0}^{\widehat{P}}$ is Zariski-dense in $\mathbb{K}^{\widehat{P}}$. (Thus we do not lose any generality from only considering zero-free labellings. The image $R^{2} f$ is not well-defined if $f$ is not zero-free anyway.)

(b) Two zero-free $\mathbb{K}$-labellings $f \in \mathbb{K}^{\widehat{P}}$ and $g \in \mathbb{K}^{\widehat{P}}$ are said to be homogeneously equivalent if and only if they satisfy one of the following two equivalent conditions:

Condition 1: For every $i \in\{0,1, \ldots, n+1\}$ and any $x \in \widehat{P}_{i}$ and $y \in \widehat{P}_{i}$, we have $\frac{f(x)}{f(y)}=\frac{g(x)}{g(y)}$.

Condition 2: There exists an $(n+2)$-tuple $\left(a_{0}, a_{1}, \ldots, a_{n+1}\right) \in\left(\mathbb{K}^{\times}\right)^{n+2}$ such that every $x \in \widehat{P}$ satisfies $g(x)=a_{\operatorname{deg} x} \cdot f(x)$.

It is clear that being homogeneously equivalent is an equivalence relation on the set $\mathbb{K}_{\neq 0}^{\widehat{P}}$ of all zerofree $\mathbb{K}$-labellings. Let $\overline{\mathbb{K}^{\widehat{P}}}$ be the set of all equivalence classes of this relation. Let $\pi: \mathbb{K}_{\neq 0}^{\widehat{P}} \rightarrow \overline{\mathbb{K}^{\widehat{P}}}$ be the canonical projection map. The set $\overline{\mathbb{K}^{\widehat{P}}}$ has an obvious structure of an algebraic variety, since it is 
isomorphic to a product of projective spaces (specifically, $\prod_{i=0}^{n+1} \mathbb{P}\left(\mathbb{K}^{\widehat{P}_{i}}\right)$ ). This allows us to talk of Zariski topology and rational maps to and from this set. The map $\pi$ itself becomes a dominant rational map $\pi: \mathbb{K}^{\widehat{P}} \rightarrow \widehat{\mathbb{K}^{\widehat{P}}}$.

Birational rowmotion and the $R_{i}$ 's respect homogeneous equivalence:

Corollary 15. Let $P$ be an n-graded poset. Let $i \in\{1,2, \ldots, n\}$. If $f \in \mathbb{K}^{\widehat{P}}$ and $g \in \mathbb{K}^{\widehat{P}}$ are two homogeneously equivalent zero-free $\mathbb{K}$-labellings, then $R_{i} f$ is homogeneously equivalent to $R_{i} g$.

Corollary 16. Let $P$ be an n-graded poset. If $f \in \mathbb{K}^{\widehat{P}}$ and $g \in \mathbb{K}^{\widehat{P}}$ are two homogeneously equivalent zero-free $\mathbb{K}$-labellings, then $R f$ is homogeneously equivalent to $R g$.

Corollary 15 would not hold with $R_{i}$ replaced by a single toggle $T_{v}$ ! So the operators $R_{i}$ in some sense combine the features of $T_{v}$ (like being an involution) with the features of $R$ (like having a simple effect on the $\mathbf{w}_{i}(f)$ checksums, cf. Proposition 12 and respecting homogeneous equivalence, cf. Corollary 15.

Definition 17. Let $P$ be an $n$-graded poset. Because of Corollary 16 the rational map $R: \mathbb{K}^{\widehat{P}} \rightarrow \mathbb{K}^{\widehat{P}}$ descends (through the projection $\pi: \mathbb{K}^{\widehat{P}} \rightarrow \overline{\mathbb{K}^{\widehat{P}}}$ ) to a partial map $\mathbb{K}^{\widehat{P}} \rightarrow \overline{\mathbb{K}^{\widehat{P}}}$. That is, there exists a partial map $\bar{R}: \overline{\mathbb{K}^{\widehat{P}}} \rightarrow \overline{\mathbb{K}^{\widehat{P}}}$ such that $\pi \circ R=\bar{R} \circ \pi$.

We could similarly define maps $\overline{R_{i}}: \overline{\mathbb{K}^{\widehat{P}}} \rightarrow \overline{\mathbb{K}^{\widehat{P}}}$ from the maps $R_{i}$.

Now, given a $\mathbb{K}$-labelling $f$ of an $n$-graded poset $P$, we can construct three images of it: the value $f(0)$ at the global minimum 0 , the $(n+1)$-tuple $\left(\mathbf{w}_{0}(f), \mathbf{w}_{1}(f), \ldots, \mathbf{w}_{n}(f)\right)$, and the projection $\pi(f)$ of $f$ on $\overline{\mathbb{K}^{\widehat{P}}}$. These three images behave like independent subsystems under birational rowmotion: The value $f(0)$ stays unchanged, the $(n+1)$-tuple is cyclically shifted (Proposition 13 , and the projection $\pi(f)$ undergoes the map $\bar{R}$. The thing that makes this observation really useful is the fact that these three images more or less determine $f$ (almost always), whence properties of the original map $R$ can be deduced by considering these three subsystems:

Proposition 18. Let $P$ be an n-graded poset. Let $f$ and $g$ be two zero-free $\mathbb{K}$-labellings in $\mathbb{K}^{\widehat{P}}$ such that $\left(\mathbf{w}_{0}(f), \mathbf{w}_{1}(f), \ldots, \mathbf{w}_{n}(f)\right)=\left(\mathbf{w}_{0}(g), \mathbf{w}_{1}(g), \ldots, \mathbf{w}_{n}(g)\right)$ and such that no $i \in\{0,1, \ldots, n\}$ satisfies $\mathbf{w}_{i}(f)=0$. Also assume that $\pi(f)=\pi(g)$ and $f(0)=g(0)$. Then, $f=g$.

Proposition 18 is easily proven by reconstructing $f$ and $g$ "bottom-up" along $\widehat{P}$.

Definition 19. Let $S$ be a set. The $\operatorname{order} \operatorname{ord} \varphi$ of a partial map $\varphi: S \rightarrow S$ is defined to be the smallest positive integer $k$ satisfying $\varphi^{k}=\mathrm{id}_{S}$, if such a positive integer $k$ exists, and $\infty$ otherwise. (This notion is quite well-behaved when $\varphi$ is a dominant rational map, and this will be the case in our situations.)

As a consequence of Proposition 18, we have ([GrRo13, §7]):

Proposition 20. Let $P$ be an $n$-graded poset. Then, ord $R=\operatorname{lcm}(n+1, \operatorname{ord} \bar{R})$. (Here, $\operatorname{lcm}(n+1, \infty)$ is to be understood as $\infty$.)

\section{Skeletal posets}

We are now going to compute the order of $R_{P}$ for a first large class of posets $P$, which we call skeletal posets. This class contains, among other posets, all graded rooted forests (see below for more details). 
Definition 21. Let $P$ be a finite poset. We denote the maps $R$ and $\bar{R}$ by $R_{P}$ and $\bar{R}_{P}$, respectively, so as to make their dependence on $P$ explicit.

Definition 22. Let $P$ and $Q$ be two $n$-graded posets. We denote by $P Q$ the disjoint union of the posets $P$ and $Q$. (This disjoint union is denoted by $P+Q$ in [Stan11, §3.2]. Its poset structure is defined in such a way that any element of $P$ and any element of $Q$ are incomparable.) Clearly, $P Q$ is again an $n$-graded poset.

Definition 23. Let $P$ be an $n$-graded poset. Let $k \in\{1,2,3, \ldots\}$. We denote by $B_{k} P$ the result of adding $k$ new elements to the poset $P$, and declaring these $k$ elements to be smaller than each of the old elements of $P$ (but incomparable with each other). Clearly, $B_{k} P$ is an $(n+1)$-graded poset. A poset $B_{k}^{\prime} P$ is defined in the same way as $B_{k} P$ but with the new elements being higher rather than smaller than the rest.

Definition 24. We define the class of skeletal posets inductively by means of the following axioms:

- The empty poset is skeletal.

- If $P$ is an $n$-graded skeletal poset and $k \in\{1,2,3, \ldots\}$, then the posets $B_{k} P$ and $B_{k}^{\prime} P$ are skeletal.

- If $n$ is a positive integer and $P$ and $Q$ are two $n$-graded skeletal posets, then the poset $P Q$ is skeletal.

Notice that every skeletal poset is graded. Also, every graded rooted forest (seen as a poset by declaring each node smaller than its children) is a skeletal poset. (Indeed, every such forest can be constructed from $\varnothing$ using merely the operations $P \mapsto B_{1} P$ and $(P, Q) \mapsto P Q$.) Similarly, every graded rooted arborescence (same as forest but each node is higher than its children) is a skeletal poset.

Skeletal posets turn out to be the simplest nontrivial class of posets on which birational rowmotion can be shown to have finite order:

Proposition 25. Let $P$ be a skeletal poset. Then, ord $\left(R_{P}\right)$ and ord $\left(\bar{R}_{P}\right)$ are finite.

The proof of this proposition ([GrRo13, §9]) follows the inductive definition of skeletal posets, making use of several facts about $R$ and $\bar{R}$. The simplest of these facts is:

Proposition 26. Let $P$ and $Q$ be two n-graded posets. Then, $\operatorname{ord}\left(R_{P Q}\right)=\operatorname{ord}\left(\bar{R}_{P Q}\right)=\operatorname{lcm}\left(\operatorname{ord}\left(R_{P}\right), \operatorname{ord}\left(R_{Q}\right)\right)$.

It is a bit trickier but still not very difficult to track the effect of $B_{k}$ on the order of $\bar{R}$ :

Proposition 27. Let $P$ be an n-graded poset.

(a) We have ord $\left(\bar{R}_{B_{1} P}\right)=$ ord $\left(\bar{R}_{P}\right)$.

(b) For every integer $k>1$, we have ord $\left(\bar{R}_{B_{k} P}\right)=\operatorname{lcm}\left(2\right.$, ord $\left.\left(\bar{R}_{P}\right)\right)$.

An exact analogue of this holds for $B_{1}^{\prime} P$ and $B_{k}^{\prime} P$ instead of $B_{1} P$ and $B_{k} P$.

Using Propositions 26, 27 (along with its analogue) and 20, it is easy to give an inductive proof of Proposition 25 and even recursively compute (not just bound from above) the orders of $R_{P}$ and $\bar{R}_{P}$ for any skeletal poset $P$ without doing any computations in $\mathbb{K}$. (This also shows that the orders of $R_{P}$ and $\bar{R}_{P}$ don't depend on the base field $\mathbb{K}$ as long as $\mathbb{K}$ is infinite and $P$ is skeletal.) For example, in the case of forests and trees, the following bound is established by induction (exact values for orders depend on the structure of the forest):

Corollary 28. Let $P$ be an $n$-graded poset. Assume that $P$ is a forest (oriented in either way). Then, ord $\left(R_{P}\right) \mid \operatorname{lcm}(1,2, \ldots, n+1)$. Moreover, if $P$ is a tree, then ord $\left(\bar{R}_{P}\right) \mid \operatorname{lcm}(1,2, \ldots, n)$. 


\section{Interlude: Classical rowmotion on skeletal posets}

The above results concerning birational rowmotion on skeletal posets suggest the question of what can be said about classical rowmotion (Definition 1) on this class of posets. Indeed, while the classical (as opposed to the birational) rowmotion map has been the object of several studies [StWi11], [CaFl95], [BrSchr74], it seems that this rather simple case has never been explicitly studied before.

Two convenient advantages of the classical setup are that we don't have to worry about denominators becoming zero, so our maps are actual maps rather than partial maps, and that we don't have to pass to $\widehat{P}$. Other than this, most of the theory developed above for birational rowmotion carries over to the case of classical rowmotion (maybe not surprisingly seeing that classical rowmotion can be interpreted as a "tropical limit" of birational rowmotion), enabling us to formulate analogues for Corollary 28, Propositions 27, 26, 25 and many of the results stated earlier. We leave the precise statements to [GrRo13, §10], while only hinting at the appropriate replacements for homogeneous equivalence and the $\mathbf{w}_{i}(f)$ 's here.

If $P$ is an $n$-graded poset and $S \in J(P)$, and $i \in\{0,1, \ldots, n\}$, then $\mathbf{w}_{i}(S)$ denotes the integer $\left\{1\right.$, if $P_{i} \subseteq S$ and $P_{i+1} \cap S=\varnothing$

$\{0$, otherwise

Here, we are using the notation $P_{j}$ for the subset $\operatorname{deg}^{-1}(\{j\})$ of $P$; this subset is empty if $j=0$ and also empty if $j=n+1$.

These numbers $\mathbf{w}_{i}(S)$ don't carry much information, being 0 for "most" order ideals. Nevertheless, they "do the trick" in the classical rowmotion case.

To define the analogue of homogeneous equivalence, we introduce one more notation. An order ideal of an $n$-graded poset $P$ is said to be level if and only if it has the form $P_{1} \cup P_{2} \cup \ldots \cup P_{i}$ for some $i \in\{0,1, \ldots, n\}$. An $n$-graded poset has precisely $n+1$ level orders, and these form an orbit under classical rowmotion $\mathbf{r}$. Two order ideals $S$ and $T$ of $P$ are said to be homogeneously equivalent if and only if either both $S$ and $T$ are level or we have $S=T$. This is clearly an equivalence relation, and we can define $\overline{J(P)}$ as the set of equivalence classes of elements of $J(P)$ modulo this relation. Let $\pi$ denote the canonical projection $J(P) \rightarrow \overline{J(P)}$. It turns out that this notion of homogeneous equivalence makes the analogues of most of our results true, most crucially that of Proposition 18

Proposition 29. Let $P$ be an $n$-graded poset. Let $S$ and $T$ be two order ideals of $P$ such that $\left(\mathbf{w}_{0}(S), \mathbf{w}_{1}(S), \ldots, \mathbf{w}_{n}(S)\right)=\left(\mathbf{w}_{0}(T), \mathbf{w}_{1}(T), \ldots, \mathbf{w}_{n}(T)\right)$ and $\pi(S)=\pi(T)$. Then, $S=T$.

It is now straightforward to define a map $\overline{\mathbf{r}}: \overline{J(P)} \rightarrow \overline{J(P)}$ in the same way as we defined a map $\bar{R}: \overline{\mathbb{K}^{\widehat{P}}} \rightarrow \overline{\mathbb{K}^{\widehat{P}}}$. This enables an analogue of Proposition 20 and of all the results we stated about skeletal posets. The analogy is strong enough to actually obtain an equality of orders:

Proposition 30. Let $P$ be a skeletal poset. Then, ord $\left(R_{P}\right)=\operatorname{ord}\left(\mathbf{r}_{P}\right)$ and $\operatorname{ord}\left(\bar{R}_{P}\right)=\operatorname{ord}\left(\overline{\mathbf{r}}_{P}\right)$.

This proposition does not generalize to arbitrary graded posets.

We notice that for graded forests, we can give the order of classical (and thus also birational) rowmotion explicitly:

Proposition 31. Let $n \in \mathbb{N}$. Let $P$ be an $n$-graded poset. Assume that $P$ is a rooted forest (made into a poset by having every node smaller than its children). Notice that $\left|\widehat{P}_{i}\right| \leq\left|\widehat{P}_{i+1}\right|$ for every $i \in$ $\{0,1, \ldots, n-1\}$. Then,

$$
\operatorname{ord}\left(\overline{\mathbf{r}}_{P}\right)=\operatorname{lcm}\left\{n-i|i \in\{0,1, \ldots, n-1\} ;| \widehat{P}_{i}|<| \widehat{P}_{i+1} \mid\right\}
$$


(Of course, ord $\left(\mathbf{r}_{P}\right)$ can now be computed by ord $\left(\mathbf{r}_{P}\right)=\operatorname{lcm}\left(n+1, \operatorname{ord}\left(\overline{\mathbf{r}}_{P}\right)\right)$.)

\section{The rectangle: statements of the results}

Definition 32. Let $p$ and $q$ be two positive integers. The poset $\{1,2, \ldots, p\} \times\{1,2, \ldots, q\}$ with order defined componentwise (that is, $(i, k) \leq\left(i^{\prime}, k^{\prime}\right)$ if and only if $\left(i \leq i^{\prime}\right.$ and $\left.k \leq k^{\prime}\right)$ ) will be denoted by Rect $(p, q)$ and called the $p \times q$-rectangle. It is a $(p+q-1)$-graded poset, with $\operatorname{deg}((i, k))=i+k-1$ for all $(i, k) \in \operatorname{Rect}(p, q)$.

The following two theorems ([GrRo13, §11]) were conjectured by James Propp and Tom Roby:

Theorem 33. We have ord $\left(R_{\operatorname{Rect}(p, q)}\right)=p+q$.

Theorem 34. Let $f \in \mathbb{K}^{\widehat{\operatorname{Rect}(p, q)}}$. Assume that $R_{\operatorname{Rect}(p, q)}^{\ell} f$ is well-defined for every $\ell \in\{0,1, \ldots, i+k-1\}$. Let $(i, k) \in \operatorname{Rect}(p, q)$. Then,

$$
f((p+1-i, q+1-k))=\frac{f(0) f(1)}{\left(R_{\operatorname{Rect}(p, q)}^{i+k-1} f\right)((i, k))} .
$$

Theorem 34 is referred to as "reciprocal symmetry property" in [EiPr13].

Notice that Proposition 20 yields that $p+q \mid \operatorname{ord}\left(R_{\operatorname{Rect}(p, q)}\right)$, so all that needs to be done in order to verify Theorem 33 is showing that $R_{\operatorname{Rect}(p, q)}^{p+q}=\mathrm{id}$.

\section{The crux of the proof}

We will only sketch the skeleta of the proofs of Theorems 33 and 34, leaving the details to [GrRo13. $\S 11-\S 16]$.

First of all, for the sake of the proofs, it can be WLOG assumed that $f(0)=f(1)=1$ (indeed, the general case can be reduced to this case by applying Proposition 20). We now define a (rational) parametrization of the space of labellings $f \in \mathbb{K}^{\operatorname{Rect}(p, q)}$ satisfying $f(0)=f(1)=1$ by certain matrices:

Definition 35. Let $A \in \mathbb{K}^{u \times v}$ be a $u \times v$-matrix for some nonnegative integers $u$ and $v$.

(a) For every $i \in\{1,2, \ldots, v\}$, let $A_{i}$ denote the $i$-th column of $A$.

(b) Moreover, we extend this definition to all $i \in \mathbb{Z}$ as follows: For every $i \in \mathbb{Z}$, let

$$
A_{i}=(-1)^{(u-1)\left(i-i^{\prime}\right) / v} \cdot A_{i^{\prime}}
$$

where $i^{\prime}$ is the element of $\{1,2, \ldots, v\}$ which is $\equiv i$ modulo $v$. (Thus, $A_{v+i}=(-1)^{u-1} A_{i}$ for every $i \in \mathbb{Z}$. Consequently, the sequence $\left(A_{i}\right)_{i \in \mathbb{Z}}$ is periodic with period dividing $2 v$, and if $u$ is odd, the period also divides $v$.)

(c) For any four integers $a, b, c$ and $d$ satisfying $a \leq b$ and $c \leq d$, we let $A[a: b \mid c: d]$ be the matrix whose columns (from left to right) are $A_{a}, A_{a+1}, \ldots, A_{b-1}, A_{c}, A_{c+1}, \ldots, A_{d-1}$. (This matrix has $b-a+d-c$ columns ${ }^{(\mathrm{i})}$ ) When $b-a+d-c=u$ (note: not just $b-a+d-c \equiv u \bmod v$ ), then this matrix $A[a: b \mid c: d]$ is a square matrix, and thus has a determinant $\operatorname{det}(A[a: b \mid c: d])$.

\footnotetext{
(i) It is not always a submatrix of $A$. Its columns are columns of $A$ multiplied with 1 or -1 ; they can appear several times and need not appear in the same order as they appear in $A$.
} 
Example 36. If $A$ is the $2 \times 3$-matrix $\left(\begin{array}{lll}3 & 5 & 7 \\ 4 & 1 & 9\end{array}\right)$, then Definition 35 (b) yields $A_{5}=(-1)^{(2-1)(5-2) / 3}$. $A_{2}=-A_{2}=-\left(\begin{array}{l}5 \\ 1\end{array}\right)=\left(\begin{array}{l}-5 \\ -1\end{array}\right)$ and $A_{-4}=(-1)^{(2-1)((-4)-2) / 3} \cdot A_{2}=A_{2}=\left(\begin{array}{l}5 \\ 1\end{array}\right)$.

Definition 37. Let $p$ and $q$ be two positive integers. Let $A \in \mathbb{K}^{p \times(p+q)}$. Let $j \in \mathbb{Z}$.

We define a map $\operatorname{Grasp}_{j} A \in \mathbb{K}^{\operatorname{Rect}(p, q)}$ by

$$
\begin{aligned}
\left(\operatorname{Grasp}_{j} A\right)((i, k))=\frac{\operatorname{det}(A[j+1: j+i \mid j+i+k-1: j+p+k])}{\operatorname{det}(A[j: j+i \mid j+i+k: j+p+k])} \\
\text { for every }(i, k) \in \operatorname{Rect}(p, q)=\{1,2, \ldots, p\} \times\{1,2, \ldots, q\}
\end{aligned}
$$

(this is well-defined for a Zariski-generic $A$ ). This map $\operatorname{Grasp}_{j} A$ will be considered as a $\mathbb{K}$-labelling of Rect $(p, q)$, by setting its values at 0 and 1 to be 1 .

The notation Grasp is meant to convey "Grassmannian parametrization", as we will parametrize (generic) labellings on Rect $(p, q)$ by matrices via this map Grasp ${ }_{0}$. While we have defined Grasp ${ }_{j}$ as a map from the matrix space $\mathbb{K}^{p \times(p+q)}$, it actually factors through the Grassmannian, because it is easy to see that Grasp $_{j} A$ is invariant under row transformations of $A$. The idea of using a ratio of determinants as in (2) goes back to Volkov's proof [Volk06] of the Zamolodchikov periodicity conjecture.

Example 38. If $p=2, q=2$ and $A=\left(\begin{array}{cccc}a_{11} & a_{12} & a_{13} & a_{14} \\ a_{21} & a_{22} & a_{23} & a_{24}\end{array}\right)$, then

$$
\left(\operatorname{Grasp}_{0} A\right)((1,1))=\frac{\operatorname{det}(A[1: 1 \mid 1: 3])}{\operatorname{det}(A[0: 1 \mid 2: 3])}=\frac{\operatorname{det}\left(\begin{array}{cc}
a_{11} & a_{12} \\
a_{21} & a_{22}
\end{array}\right)}{\operatorname{det}\left(\begin{array}{cc}
-a_{14} & a_{12} \\
-a_{24} & a_{22}
\end{array}\right)}=\frac{a_{11} a_{22}-a_{12} a_{21}}{a_{12} a_{24}-a_{14} a_{22}}
$$

and

$$
\left(\operatorname{Grasp}_{1} A\right)((1,2))=\frac{\operatorname{det}(A[2: 2 \mid 3: 5])}{\operatorname{det}(A[1: 2 \mid 4: 5])}=\frac{\operatorname{det}\left(\begin{array}{cc}
a_{13} & a_{14} \\
a_{23} & a_{24}
\end{array}\right)}{\operatorname{det}\left(\begin{array}{ll}
a_{11} & a_{14} \\
a_{21} & a_{24}
\end{array}\right)}=\frac{a_{13} a_{24}-a_{14} a_{23}}{a_{11} a_{24}-a_{14} a_{21}} .
$$

Remark 39. Let $p$ and $q$ be two positive integers. Let $A \in \mathbb{K}^{p \times(p+q)}$ be a Zariski-generic matrix. Then, $\operatorname{Grasp}_{j} A=\operatorname{Grasp}_{p+q+j}$ A for every $j \in \mathbb{Z}$.

Remark 40. Let $p$ and $q$ be two positive integers. Let $A \in \mathbb{K}^{p \times(p+q)}$ be a Zariski-generic matrix. Let $j \in \mathbb{Z}$. Then,

$$
\left(\operatorname{Grasp}_{j} A\right)((i, k))=\frac{1}{\left(\operatorname{Grasp}_{j+i+k-1} A\right)((p+1-i, q+1-k))} .
$$

Now, let us state the two facts which will combine to a proof of Theorem 33 .

Proposition 41. Let $p$ and $q$ be two positive integers. Let $A \in \mathbb{K}^{p \times(p+q)}$ be a Zariski-generic matrix. Let $j \in \mathbb{Z}$. Then, $\operatorname{Grasp}_{j} A=R_{\text {Rect }(p, q)}\left(\operatorname{Grasp}_{j+1} A\right)$. 
Proposition 42. Let $p$ and $q$ be two positive integers. For almost every (in the Zariski sense) reduced labelling $f \in \mathbb{K}^{\mathrm{Rect}(p, q)}$, there exists a matrix $A \in \mathbb{K}^{p \times(p+q)}$ satisfying $f=\operatorname{Grasp}_{0} A$.

Once these propositions are proven, Theorems 33 and 34 easily follow (details are found in [GrRo13]).

We will not dwell on the proof of Proposition 42 it being a rather technical "triangularity" argument (in the sense that the construction of $f$ from $A$ can be done by recursively eliminating the values of $f$ from a triangular, even if non-linear, system of equations). The details are found in [GrRo13, §15].

The proof of Proposition 41] is computational ([GrRo13, §14]). It uses basic properties of determinants as well as what we call the Plücker-Ptolemy relation:

Theorem 43. Let $A \in \mathbb{K}^{u \times v}$ be $a u \times v$-matrix for some nonnegative integers $u$ and $v$. Let $a, b, c$ and $d$ be four integers satisfying $a \leq b$ and $c \leq d$ and $b-a+d-c=u-2$. Then,

$$
\begin{aligned}
& \operatorname{det}(A[a-1: b \mid c: d+1]) \cdot \operatorname{det}(A[a: b+1 \mid c-1: d]) \\
& +\operatorname{det}(A[a: b \mid c-1: d+1]) \cdot \operatorname{det}(A[a-1: b+1 \mid c: d]) \\
& =\operatorname{det}(A[a-1: b \mid c-1: d]) \cdot \operatorname{det}(A[a: b+1 \mid c: d+1]) .
\end{aligned}
$$

This theorem is a particular case of the Plücker relations, but can also be proven in a very pedestrian way. It generalizes the well-known Dodgson condensation identity.

\section{Results for other posets}

In general, finite posets $P$ having a finite ord $\left(R_{P}\right)$ seem to be an exception rather than the rule. For instance, ord $\left(R_{P}\right)=\infty$ when $P$ is the Boolean lattice [2] $\times[2] \times[2]$, as well as for some other relatively simple posets (even some "slices" of the otherwise well-behaved rectangles Rect $(p, q)$ ); see [GrRo13, $\S 20]$. We have not found any example of a non-graded poset $P$ for which ord $\left(R_{P}\right)<\infty$.

There are, however, some more posets $P$ with finite ord $\left(R_{P}\right)$ which are not covered by the skeletal and rectangle cases. Some results are:

1. If $P$ is the subposet of Rect $(p, p)$ consisting of all $(i, k)$ satisfying $i \leq k$ (that is, the left half of the $p \times p$-square, including the diagonal, speaking in terms of Hasse diagrams), then ord $\left(R_{P}\right)=2 p$. This is proven ([GrRo13, §17]) using a folding argument and the above results for rectangles.

2. If $P$ is the subposet of $\operatorname{Rect}(p, p)$ consisting of all $(i, k)$ satisfying $i+k>p+1$ (that is, the upper half of the $p \times p$-square, excluding the diagonal, speaking in terms of Hasse diagrams), then ord $\left(R_{P}\right)=2 p$ (if $p>2$ ), and moreover, $R_{P}^{p}$ amounts to reflecting the labelling across the vertical axis. This, again, follows using (subtler) folding arguments ([GrRo13, §18]) from the rectangle case. Analogous facts hold for the lower half of the square.

3. If $P$ is the subposet of Rect $(p, p)$ consisting of all $(i, k)$ satisfying both $i+k>p+1$ and $i \leq k$ (this is a rectangular triangle, in Hasse-diagram language), then we conjecture that ord $\left(R_{P}\right)=p$ (for $p>1$ ). We have a proof of this for $p$ odd ([GrRo13, §19]); Nathan Williams has suggested a further conjectural generalization to trapezoid-shaped posets.

4. It seems that root posets in "coincidental types" $\left(A, B, H_{3}\right)$ and minuscule heaps provide examples of posets $P$ with finite ord $\left(R_{P}\right)$. More precisely, the positive root poset in type $A_{n}$ is the poset $P$ 
of item 1 above (for $p=n+1$ ), and the positive root poset in type $B_{n}$ is the poset $P$ of item 3 above (for $p=2 n$ ). The "positive root poset in type $H_{3}$ " (in Cuntz's sense) satisfies ord $\left(R_{P}\right)=10$. See [GrRo13, §21] for a brief review of this (largely mysterious) connection.

\section{References}

[AKSch12] Arvind Ayyer, Steven Klee, Anne Schilling, Combinatorial Markov chains on linear extensions, Journal of Algebraic Combinatorics, September 2013, DOI 10.1007/s10801-0130470-9. Also available at arXiv:1205.7074v3,

[AST11] D. Armstrong, C. Stump and H. Thomas, A uniform bijection between nonnesting and noncrossing partitions, Trans. Amer. Math. Soc. 365 (2013), pp. 4121-4151, DOI 10.1090/S0002-9947-2013-05729-7. A preprint is available at arXiv:1101.1277v2.

[BrSchr74] Andries E. Brouwer and A. Schrijver, On the period of an operator, defined on antichains, Math. Centr. report ZW24, Amsterdam (Jun. 1974).

[CaF195] Peter J. Cameron, Dmitry G. Fon-der-Flaass, Orbits of Antichains Revisited, European Journal of Combinatorics, vol. 16, Issue 6, November 1995, pp. 545-554.

[EiPr13] David Einstein, James Propp, Combinatorial, piecewise-linear, and birational homomesy for products of two chains, arXiv:1310.5294v1 (preliminary version), October 20, 2013.

[Flaa93] Dmitry G. Fon-der-Flaass, Orbits of Antichains in Ranked Posets, European Journal of Combinatorics, vol. 14, Issue 1, January 1993, pp. 17-22.

[GrRo13] Darij Grinberg and Tom Roby, Iterative properties of birational rowmotion, preprint Citations refer to version 2.0 (arXiv:1402.6178v2 (not yet online, see MIT link)).

[Pan08] D.I. Panyushev, On orbits of antichains of positive roots, Europ. J. Combin. 30 (2009), no. 2, pp. 586-594. Also available at arXiv:0711.3353v2.

[PrRo13] James Propp and Tom Roby, Homomesy in products of two chains, arXiv:1310.5201v2

[Sage08] The Sage-Combinat community, Sage-Combinat: enhancing Sage as a toolbox for computer exploration in algebraic combinatorics, 2008. http://combinat.sagemath.org

[Stan11] Richard Stanley, Enumerative Combinatorics, volume 1, 2nd edition 2011.

[Stan86] Richard Stanley, Two Poset Polytopes, Discrete \& Computational Geometry, 1986, Volume 1, Issue 1, pp. 9-23.

[StWi11] Jessica Striker, Nathan Williams, Promotion and Rowmotion, European Journal of Combinatorics 33 (2012), pp. 1919-1942, DOI 10.1016/j.ejc.2012.05.003. Also available as arXiv:1108.1172v3.

[Volk06] Alexandre Yu. Volkov, On the Periodicity Conjecture for Y-systems, Commun. Math. Phys. 276 (2007), pp. 509-517, DOI 10.1007/s00220-007-0343-y. A preprint is available as arXiv:hep-th/0606094v1. 